Check for updates

Cite this: RSC Adv., 2018, 8, 32252

\section{Synthesis of a lignin-based phosphorus-containing flame retardant and its application in polyurethane}

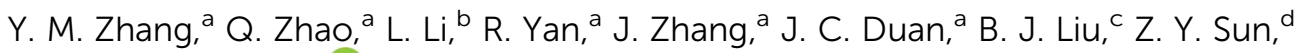 \\ M. Y. Zhang, ${ }^{a}$ W. Hu (D) ${ }^{* a}$ and N. N. Zhang*a
}

\begin{abstract}
In this work, new lignin-based flame retardant LHDs were successfully synthesized through the reaction between lignin, 9,10-dihydro-9-oxa-10-phosphaphenanthrene-10-oxide (DOPO) and hexamethylene diisocyanate (HDI). The chemical structure of LHD was characterized by FTIR, ${ }^{1} \mathrm{H}$ NMR, ${ }^{31} \mathrm{P}$ NMR. The thermal stability of LHD was studied by TGA. The results showed that the residual carbon content of L15HD ( $15 \%$ of lignin in LHD) at $600{ }^{\circ} \mathrm{C}$ reached $16.55 \%$, indicating that this prepared flame retardant can be a type of good char forming agent. LHDs were then applied to prepare flame-retardant lignin-based polyurethane (FLPU). Lignin-based polyurethane (LPU) was synthesized by the reaction between lignin, polyethylene glycol 200 (PEG 200) and hexamethylene diisocyanate (HDI). The limiting oxygen index (LOI) value of the FLPU reached $30.2 \%$ when the addition content of L15HD (15\% lignin in LHD) in $\mathrm{L}_{20} \mathrm{PU}(20 \%$ lignin in LPU) was $25 \%$, exhibiting excellent flameretardant properties. Scanning electron microscopy (SEM) analysis of the FLPU char residual showed that there was a continuous dense outer carbon layer on the residue surface, and the inner carbon layer had many expansion bubbles, indicating the LHDs have an excellent flame retardant effect for PU. In addition, FLPU presented better hardness and adhesion than PU. The hardness of $F_{\llcorner 15-25} L_{20} \mathrm{PU}$ (lignin content in LPU was $20 \%$, and added content of L15HD in LPU was $25 \%$ ) reached $4 \mathrm{H}$, and its adhesion was 0 . These excellent properties illustrated that the LHDs are ideal flame retardants and reinforcing agents for LPU because of the co-curing and strong interface between LHD and LPU.
\end{abstract}

Received 30th June 2018

Accepted 31st August 2018

DOI: 10.1039/c8ra05598j

rsc.li/rsc-advances

\section{Introduction}

Lignin, a complex natural aromatic polymer, is the second most abundant biomass next to cellulose. At present, lignin is widely available as a major by-product of the pulp and paper industries as well as bio-refineries. ${ }^{1-3}$ It is composed of three different types of phenylpropane units, $p$-coumaryl alcohol, coniferyl alcohol, and sinapyl alcohol.,4 They form a rigid, threedimensional network structure. ${ }^{5}$ With the impressive properties of renewability, biodegradability, environmental friendliness and low price, lignin has become an excellent sustainable green chemical raw material. ${ }^{2-4,6}$ Lignin has various functional groups, such as hydroxyl units (both phenolic and aliphatic), which make it easy to be modified., ${ }^{4,5}$. On the other hand, thanks to its aromatic structure, lignin could be advantageously applied in polymeric matrices as a char promotor agent that can

${ }^{a}$ College of Chemical Engineering, Changchun University of Technology, 2055 Yan'an Street, Changchun 130012, P. R. China. E-mail: huwei@ccut.edu.cn; Fax: +86-43185717216; Tel: +86-431-85717216

${ }^{b}$ School of Life Science and Technology, Harbin Institute of Technology, Harbin 150080, P. R. China

${ }^{c}$ College of Chemistry, Jilin University, 2699 Qianjin Street, Changchun 130012, P. R. China

${ }^{d}$ State Key Laboratory of Polymer Physics and Chemistry, Changchun Institute of Applied Chemistry, Chinese Academy of Sciences, Changchun 130022, China allow some reduction of the combustion rate. As a consequence, the enormous potential of lignin in value-added products is its application as the precursor of different classes of polymeric materials, including epoxies, ${ }^{8,9}$ polyesters, ${ }^{10,11}$ phenolics $^{12}$ and polyurethanes. ${ }^{13,14}$ One strategy to broaden the practical application of lignin in industry for environmental protection and the utilization of natural resources has outstanding economic and social benefits.

Polyurethane (PU) contained urethane linkage (-NH-COO-), which was synthesized by a reaction of diisocyanate with diol (with functionality higher than 2). Polyurethane has been widely capitalized on aerospace, automotive, coatings, and other fields because of their unique merits, such as low density, high tensile strength, and high dimensional stability. ${ }^{15} \mathrm{PU}$ has rapidly grown to be one of the most diverse and widely used plastics with a continuously increasing global market. Nonetheless, diol, one of the most important compounds dependent on petrochemical resources, is crucial for the expenses of PU. There are phenol and hydroxyl groups on lignin, which could also react with the isocyanate. It is very promising to replace the fossil sources by the renewable lignin as additives or part of the reactant monomer to obtain eco-friendly bio-polyurethane.

On the other hand, the inherently high flammability of PU has restricted its application. Hence, to extend the durable applications of $\mathrm{PU}$, its flame retardant property need to be 
improved. To improve the fire-resistant property of PU is an interesting research subject. To improve the fire-resistant property of PU is an interesting research subject. Adding the flame-retardant (FR) additives into the polymers is the most popular approach to improve the flame retardancy. ${ }^{16-18}$ Many literatures had reported that lignin and its derivatives were used as flame retardants of polyurethanes and other materials.

For example, Lu et al. used lignosulfonate to substitute part of diethylene glycol (DEG) to copolymerize with isocyanate to produce lignosulfonate based rigid polyurethane (LRPU) foams. Lignosulfonate was employed as carbonization agent and combined with APP to improve the flame retardancy of LRPU. ${ }^{19}$ Gao et al. obtained liquefied lignin-based polyol by liquefying calcium lignosulphonate with a polyhydroxy alcohol mixture. And it was simultaneously incorporated with commercial flame retardant polyol, polyurethane microencapsulated ammonium polyphosphate and organically modified layered double hydroxide into rigid polyurethane foam (RPUF). The results indicated that the mechanical property, thermal performance, flame retardancy and fire behaviour of RPUF composites were improved. ${ }^{20}$ Zhu et al. prepared a flame retardant modified lignin (LPMC) containing chemically grafted phosphorusnitrogen by liquefaction, esterification and salt formation. And then the LPMC was applied to prepare polyurethane foam. The LOI value of polyurethane foam was $26.7 \%$, passing the UL94 measurement V-1 grade with no dropping. ${ }^{21}$ However, the lignin in many studies was necessary to be purified before use, and the modification process were so complicated in the experiments. Others also applied the modified lignin to polypropylene, ${ }^{22}$ polylactic acid, ${ }^{23}$ and acrylonitrile butadiene styrene, etc. ${ }^{24}$ but the properties were always not so preferable. The specific comparison of some related reports are shown in Table 1.

Nowadays, 9,10-dihydro-9-oxa-10-phosphaphenanthrene-10oxide (DOPO) and its derivatives are considered as suitable alternative to halogenated flame retardants in the light of its effective extinguishing behaviour in gas phase and condensed phase, and its environmentally friendliness. ${ }^{25}$ And so far, there was no report on the use of HDI as a bridge to link commercial lignin molecular with DOPO to produce a flame retardant with low expenses.

In this work, a lignin-based flame-retardant noted as LHD was synthesized by the reaction of DOPO, hexamethylene diisocyanate (HDI) and lignin. Diisocyanate was used as a bridge to connect lignin and DOPO. This could also be an effective way for solving the compatibility between the flame retardant and the matrix PU or lignin-based PU (LPU). The mechanical properties of the flame-retardant lignin-based polyurethane (FLPU) composites can be reinforced by LHD because it contained the same urethane structure with polyurethane and the phenyl groups in lignin. Thus, the FLPU films could contain not only the flame retardant element phosphorus but also the bioproduct lignin, which could act as the reinforcing agent and carbonizing agent. The performance of the flame retardant lignin based PU films in terms of thermal stability, flame retardancy and properties as coatings were studied in detail.

\section{Experimental}

\section{Material}

DOPO (99\%) was purchased from Zhengzhou Alpha Chemical Co., Ltd. Organosolv lignin was obtained from Yanghai Chemical Co., Ltd. Hexamethylene diisocyanate (HDI) (99\%) was provided by Macklin. PEG200 $\left(M_{\mathrm{r}}=190-210 \mathrm{~g} \mathrm{~mol}^{-1}\right)$ was brought from Tianjin Institute of Fine Chemical rehabilitation. Dibutyltin dilaurate (95\%) as a catalyst was purchased from Macklin. Triethylamine (AR) was obtained from West Long Chemical Co., Ltd. N,N-dimethylformamide (95\%, AR) was provided by Tianjin Tiantai Fine Chemicals Co. Ltd. Ethanol (AR, $98 \mathrm{v} \%$ ) was purchased from Sinopharm Chemical Reagent Co., Ltd.

Table 1 Comparison of flame retardant with lignin reported in literature

References Modified method

19 Lignosulfonate/APP

20

21 (OLDH)

1
Liquefied lignin-based polyol (LBP)/MAPP/ organically modified layered double hydroxide Lignin was chemically grafted with phosphorusnitrogen-containing groups via a liquefactionesterification-salification process to prepare ligninbased phosphate melamine compound (LPMC)

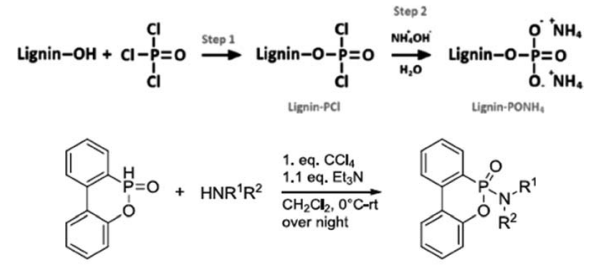

Scheme 1. Synthesis of DoPO-phosphonamidates.
Flame retardancy

The heat release rate (HRR) and total heat release (THR) was reduced; the LOI value increased to be over $30 \%$

LOI was up to $28.1 \%$; the initial degradation temperature of modified foam increased from $228{ }^{\circ} \mathrm{C}$ to $265{ }^{\circ} \mathrm{C}$, and the mass residue of foam at $900{ }^{\circ} \mathrm{C}$ increased from 0.6 to $15.4 \%$

LOI values reached to be $28.3 \%$; the UL-94 tests were achieved V- 1 rate

The samples reached V-0 in UL-94 test

BKZ tests were reached the highest to HF-2; thermal stability was improved 

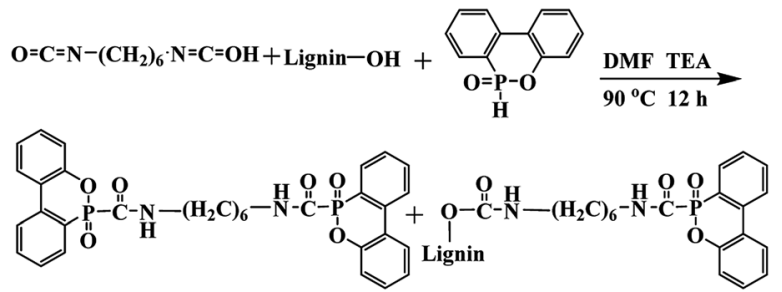

Scheme 1 Synthesis of LHDs.

\section{Synthesis of lignin-based flame retardant (LHDs)}

DOPO was dried at $80{ }^{\circ} \mathrm{C}$ overnight before use to remove water. To a three-necked round-bottom, DOPO, HDI, lignin (total hydroxyl content is $3.225 \mathrm{mmol} \mathrm{g}^{-1}$ ) at $1: 1$ molar ratio of NCO: $(-\mathrm{OH}$ and $-\mathrm{P}-\mathrm{H})$. The mass of catalyst triethylamine was $0.4 \%$ and a certain amount of DMF was added. The amount of DOPO used in all formulations was $0.1 \mathrm{~mol}$. The mixture was reacted at $90{ }^{\circ} \mathrm{C}$ for $12 \mathrm{~h}$. Then, the reaction mixture was cooled to room temperature, washed with ethanol thoroughly, and dried at $80^{\circ} \mathrm{C}$ for $12 \mathrm{~h}$ in a vacuum oven and then ground into powder. The synthesis route was shown in Scheme 1.

\section{Preparation of the flame-retarding PU composites (FLPU films)}

Polyurethanes were prepared through condensation reactions between -NCO in hexamethylene diisocyanate (HDI) and the hydroxyl groups in polyethylene glycol (PEG 200) and lignin. The lignin content was $20 \%$. PEG 200 was used to be the soft segment in polyurethane. In our work, the molar ratio of $\mathrm{NCO} /$ $\mathrm{OH}$ was 1.3 , which was calculated according to the isocyanate group from HDI and total hydroxyl content from lignin (phenolic and aliphatic group) and polyethylene glycol.

Typically, $\mathrm{F}_{\mathrm{L} 15-25} \mathrm{~L}_{20} \mathrm{PU}$ (content of lignin in the flameretardant lignin-based polyurethane was $20 \%$, content of lignin in the LHD was $15 \%$, and the mass fraction of the LHD was $25 \%$ ) was synthesized as following: $4 \mathrm{~g}$ lignin was dissolved in $30 \mathrm{ml}$ of DMF under magnetic stirring. Subsequently, $6.97 \mathrm{~g}$ PEG200 was added, then $9.03 \mathrm{~g}$ HDI and $0.4 \mathrm{~g}$ dibutyltin dilaurate was added. After stirring at $80{ }^{\circ} \mathrm{C}$ about $90 \mathrm{~min}, 6.67 \mathrm{~g}$ L15HD powder was added into the prepared polyurethane prepolymer and mixed well. Then the mixture was poured into molds carefully and cured at room temperature overnight, followed by heating at $50{ }^{\circ} \mathrm{C}, 100{ }^{\circ} \mathrm{C}$ for $12 \mathrm{~h}$, respectively. The thickness of the films were about $1 \mathrm{~mm}$.

\section{Characterization}

FTIR analysis. Fourier transform infrared (FTIR) spectra (EQUINOX55, Bruker Co., Germany) was employed to characterize the structure of LHDs using $\mathrm{KBr}$ pellets as the sample holder. The wavenumber range was set from $4000 \mathrm{~cm}^{-1}$ to $600 \mathrm{~cm}^{-1}$ with a resolution of $4 \mathrm{~cm}^{-1}$.

NMR spectra analysis. ${ }^{1} \mathrm{H}$ NMR and ${ }^{31} \mathrm{P}$ NMR spectra were obtained at room temperature on a Bruker Avance Spectrometer (400 MHz) with DMSO- $\mathrm{d}_{6}$ as solvent, and tetramethylsilane
(TMS) as the internal standard and $\mathrm{H}_{3} \mathrm{PO}_{4}(85 \%)$ as the external standard respectively.

Thermogravimetric analyses (TGA) analysis. Thermal decomposition of the different content of lignin in LHDs and different FLPU composites were studied on a Shimadzu DTG$60 / 60 \mathrm{H}$ by thermogravimetric analysis (TGA). The samples were submitted to a temperature ramp from 50 to $700{ }^{\circ} \mathrm{C}$ at a heating rate of $20{ }^{\circ} \mathrm{C} \mathrm{min}^{-1}$. All TGA experiments were performed under a nitrogen flow of nitrogen flow rate of $20 \mathrm{ml} \mathrm{min}^{-1}$.

X-ray photoelectron spectroscopy (XPS) test. The X-ray photoelectron spectroscopy (XPS) data were obtained using a PerkinElmer PHI 5300 ESCA system at $250 \mathrm{~W}(12.5 \mathrm{kV}$ at 20 $\mathrm{mA})$ under a vacuum higher than $10^{-6} \mathrm{~Pa}$. The char samples were obtained through treating in nitrogen atmosphere in the muffle furnace at different temperatures. Typical results from XPS were reproducible within $\pm 3 \%$, and the reported results are the average of three measurements.

Limiting oxygen index (LOI) test. The limiting oxygen index (LOI) test was carried out on a M606B limit oxygen index tester (Qingdao Shanfang Instrument Co., Ltd.) according to standard methods GB/T2406-2009 with the sheet size $140 \times 52 \times 1 \mathrm{~mm}$.

Scanning electron microscopy (SEM) analysis. Scanning electron microscopy (SEM) was carried out on a XL30 ESEM-FEG scanning electron microscope, in the high vacuum mode, at $15.0 \mathrm{kV}$ accelerating voltage.

Coating properties test. The prepared film was coated on the tinplate to test its flexibility, hardness, adhesion and impact resistance, based on the national standards GB/T 1731-93, GB/T 6379-1996, GB/T 9286-1998 and GB/T 1732-93, respectively.

\section{Results and discussion}

\section{Chemical characterization of lignin-based flame-retardant (LHDs)}

A lignin based flame-retardant noted as LHD was synthesized by the reaction of DOPO, hexamethylene diisocyanate (HDI) and lignin as shown in Scheme 1. Diisocyanate was used as a bridge to connect lignin and DOPO. The flame retardant prepared by the method of the present study was expected to form a conjugation $\mathrm{O}=\mathrm{C}-\mathrm{P}=\mathrm{O}$, which was helpful to increase the thermal stability and flame retardancy of the product. More $\mathrm{O}=\mathrm{C}-\mathrm{P}=\mathrm{O}$ structure was produced with the increased temperature. The chemical structure of the synthesized LHDs was characterized by FTIR, ${ }^{1} \mathrm{H}$ NMR and ${ }^{31} \mathrm{P}$ NMR.

\section{FTIR characterization of LHDs}

Fig. 1 showed the FTIR spectra of DOPO and LHDs. From the FTIR spectrum of DOPO, the peak at $2434 \mathrm{~cm}^{-1}$ was belonged to the stretching vibration of $\mathrm{P}-\mathrm{H}$ group. ${ }^{21}$ In case of LHDs, there were several absorption peaks. The peak at $3280 \mathrm{~cm}^{-1}$ was corresponded to $\mathrm{N}-\mathrm{H}$ group; the peaks at $1593 \mathrm{~cm}^{-1}$ and $1475 \mathrm{~cm}^{-1}$ was attributed to $\mathrm{P}-\mathrm{C}$, and the peak at $1200 \mathrm{~cm}^{-1}$ was related to the $\mathrm{P}=\mathrm{O}$ group, ${ }^{25}$ while the characteristic absorption peak of $\mathrm{P}-\mathrm{H}$ group in DOPO disappeared. The absorption peaks around $2932 \mathrm{~cm}^{-1}$ and $2857 \mathrm{~cm}^{-1}$ corresponded to the 


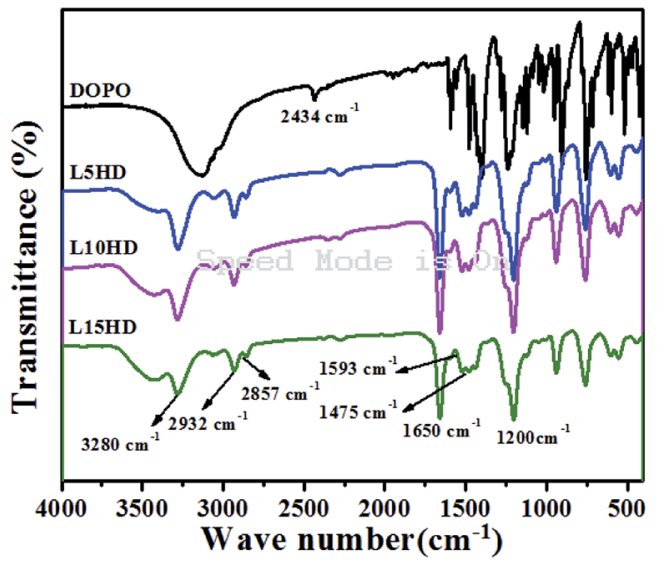

Fig. 1 FTIR spectra of DOPO and LHDs.

vibration of $-\mathrm{CH}_{2}$ in HDI. Furthermore, in the FTIR spectrum of LHDs, the peak at $1650 \mathrm{~cm}^{-1}$ was assigned as the stretching vibration of $\mathrm{C}=\mathrm{O}$ bond of HDI. ${ }^{25}$ It was indicated that LHDs have been successfully synthesized.

\section{${ }^{1} \mathrm{H}$ NMR characterization of LHDs}

The ${ }^{1} \mathrm{H}$ NMR spectra of the synthesized LHDs were presented in Fig. 2. As to DOPO, the peak at $8.83 \mathrm{ppm}$ was attributed to the proton in $\mathrm{P}-\mathrm{H}^{26}$ In the case of LHDs, the chemical shift at $8.83 \mathrm{ppm}$ disappeared and the peaks at $9.3 \mathrm{ppm}$ belonged to $\mathrm{N}-\mathrm{H}$ appeared. ${ }^{26}$ This further suggested that the LHDs was synthesized successfully. The peaks between 7.0 and $8.3 \mathrm{ppm}$ were corresponded to the protons in the benzene ring. Additionally, the peaks at 2.5-3.4 ppm were assigned to the $\mathrm{CH}_{2}$ in HDI. The two peaks at $1.1 \mathrm{ppm}$ and $1.4 \mathrm{ppm}$ should be corresponded to the $-\mathrm{CH}_{3}$ of lignin.

\section{${ }^{31} \mathrm{P}$ NMR characterization of LHDs}

The ${ }^{31} \mathrm{P}$ NMR spectra of DOPO and LHDs were described in Fig. 3. The peaks at $12.6 \mathrm{ppm}$ and $16.4 \mathrm{ppm}$ were attributed to the phosphorus atoms in DOPO group. The peak at $11.3 \mathrm{ppm}$

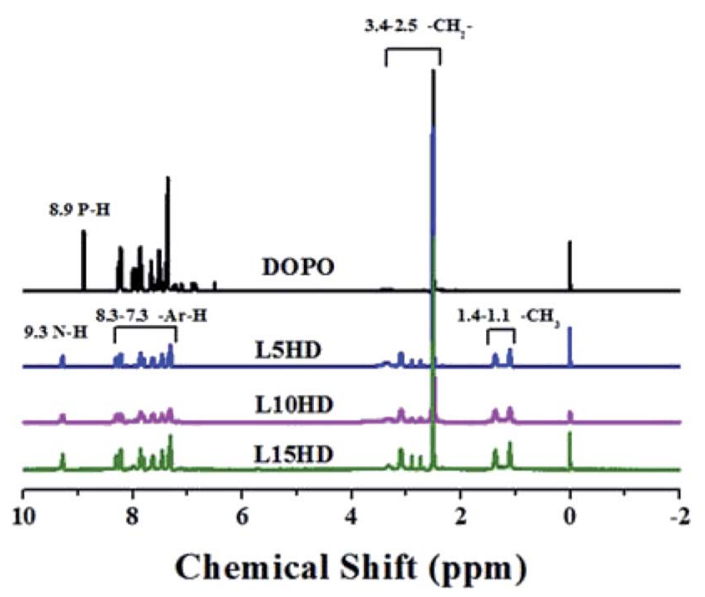

Fig. $2{ }^{1} \mathrm{H}$ NMR of DOPO and LHDs.

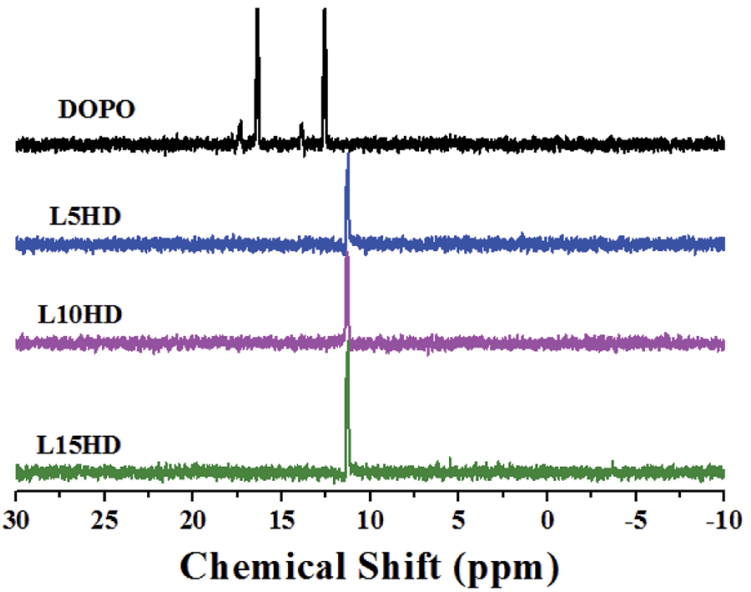

Fig. $3{ }^{31} \mathrm{P}$ NMR of DOPO and LHDs.

was assigned to the phosphorus atoms in the LHDs. All the above characterization results confirmed the chemical structure of the prepared LHDs. It was reasonable to conclude that the LHDs were successfully synthesized.

\section{TGA analysis of DOPO and LHDs}

The detailed information about thermal stability and thermal degradation behaviour of LHDs was investigated by TG analysis test. Fig. 4 shows the TGA curves of samples. The data results are summarized in Table 2.

It can be seen from Fig. 4 that DOPO started to decompose (5\% weight loss temperature $\left(T_{5}\right)$ ) at $220^{\circ} \mathrm{C}$, and there was no major residue left after $300^{\circ} \mathrm{C}$. This was consistent with another report. ${ }^{25}$ The thermal degradation temperature of lignin had a broad temperature range..$^{29,30}$ In the range of $100-170{ }^{\circ} \mathrm{C}$, the loss of water happened. The decomposition of $\beta$-aryl-alkyl-ether bond occurred between $180-230{ }^{\circ} \mathrm{C}^{31} T_{5}$ of lignin was $210{ }^{\circ} \mathrm{C}$, which was lower than that of DOPO and showed the lower thermal stability of lignin. The degradation of the propanoid side chain of lignin occured in the temperature range of 230$260{ }^{\circ} \mathrm{C}$, with the formation of low molecular weight products. The $\mathrm{C}-\mathrm{C}$ and $\beta-\beta$ linkages in the lignin cleaved at $275-350{ }^{\circ} \mathrm{C}$.

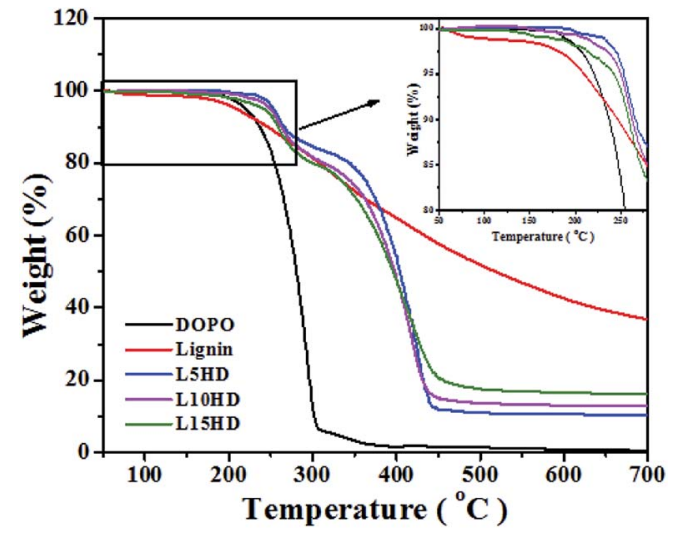

Fig. 4 TGA curves of DOPO and LHDs. 
Table 2 TGA characterization of DOPO and LHDs ${ }^{a}$

\begin{tabular}{llllrr}
\hline & & & \multicolumn{3}{c}{ Char residue $(\%)$} \\
\cline { 5 - 6 } Samples & $T_{5}\left({ }^{\circ} \mathrm{C}\right)$ & $T_{10}\left({ }^{\circ} \mathrm{C}\right)$ & $500{ }^{\circ} \mathrm{C}$ & $600{ }^{\circ} \mathrm{C}$ & $700{ }^{\circ} \mathrm{C}$ \\
\hline DOPO & 220 & 237 & 1.34 & 0.78 & 0.42 \\
Lignin & 210 & 247 & 51.89 & 42.59 & 36.72 \\
L5HD & 253 & 266 & 10.94 & 10.49 & 10.42 \\
L10HD & 250 & 262 & 13.66 & 13.00 & 12.82 \\
L15HD & 242 & 258 & 17.54 & 16.55 & 16.13
\end{tabular}

${ }^{a} T_{5}$ : initial degradation temperature (temperature at $5 \%$ weight loss). $T_{10}$ : temperature at $10 \%$ weight loss.

Above $500{ }^{\circ} \mathrm{C}$, the formation of char was resulted by the further rearrangements and condensation of the aromatic structure. These results were consistent with other reports. ${ }^{23,30,32,33}$

The flame retardant prepared by the method of the present study was expected to form a conjugation between $\mathrm{O}=\mathrm{C}-\mathrm{P}=\mathrm{O}$, which could help to increase the thermal stability of the product. This should be attributed to the beneficial $\pi-\pi$ interaction of the aromatic groups between DOPO itself and the additional benzyl of lignin. ${ }^{34}$ The $T_{5}$ and $T_{10}$ of LHDs were much higher than that of DOPO and lignin. $T_{5}$ of L5HD reached $253{ }^{\circ} \mathrm{C}$. It was shown in Table 2 that the thermal degradation temperature of LHDs gradually decreased with the increase of lignin content, which was mainly because that the lignin portion degraded at lower temperature.
Furthermore, the $\mathrm{O}=\mathrm{C}-\mathrm{P}=\mathrm{O}$ structure could act as acid source and blowing agent during combustion. And it would be thermo-oxidized, dehydrated by the acid, and to act as carbonizing agent. On the other hand, the char yield of lignin was up to $42.59 \%$ at $600{ }^{\circ} \mathrm{C}$, as shown in Table 2, indicating that it was an excellent char-forming agent owing to its high content of aromatic ring structure. ${ }^{21}$ The char was the decomposed product after the lignin was fired at high temperature. Therefore, it should be very stable. It could insulate heat and material transfer, reduce the rate of combustion of the polymer and block oxygen, which play an efficient role in flame retardancy. The ability to form char during the thermal degradation was a basic aspect of flame retardant intumescent systems. ${ }^{22,27}$

It can be observed that LHDs have higher char yield than DOPO at $600{ }^{\circ} \mathrm{C}$ as shown in Fig. 4 and Table 2. The residual carbon content increased with the lignin content. And the residual carbon content of L15HD was as high as $17.54 \%$ at $500{ }^{\circ} \mathrm{C}$, which showed high thermal stability. ${ }^{35}$ The high residual char content was attributed to the ether bonds and aromatic ring structures in lignin.

\section{X-ray photoelectron spectroscopy (XPS) test}

The L15HD sample was treated in a muffle furnace under a nitrogen atmosphere at various temperatures, and thereafter XPS measurement were measured to check the elemental composition and nature at various temperature ranges as an
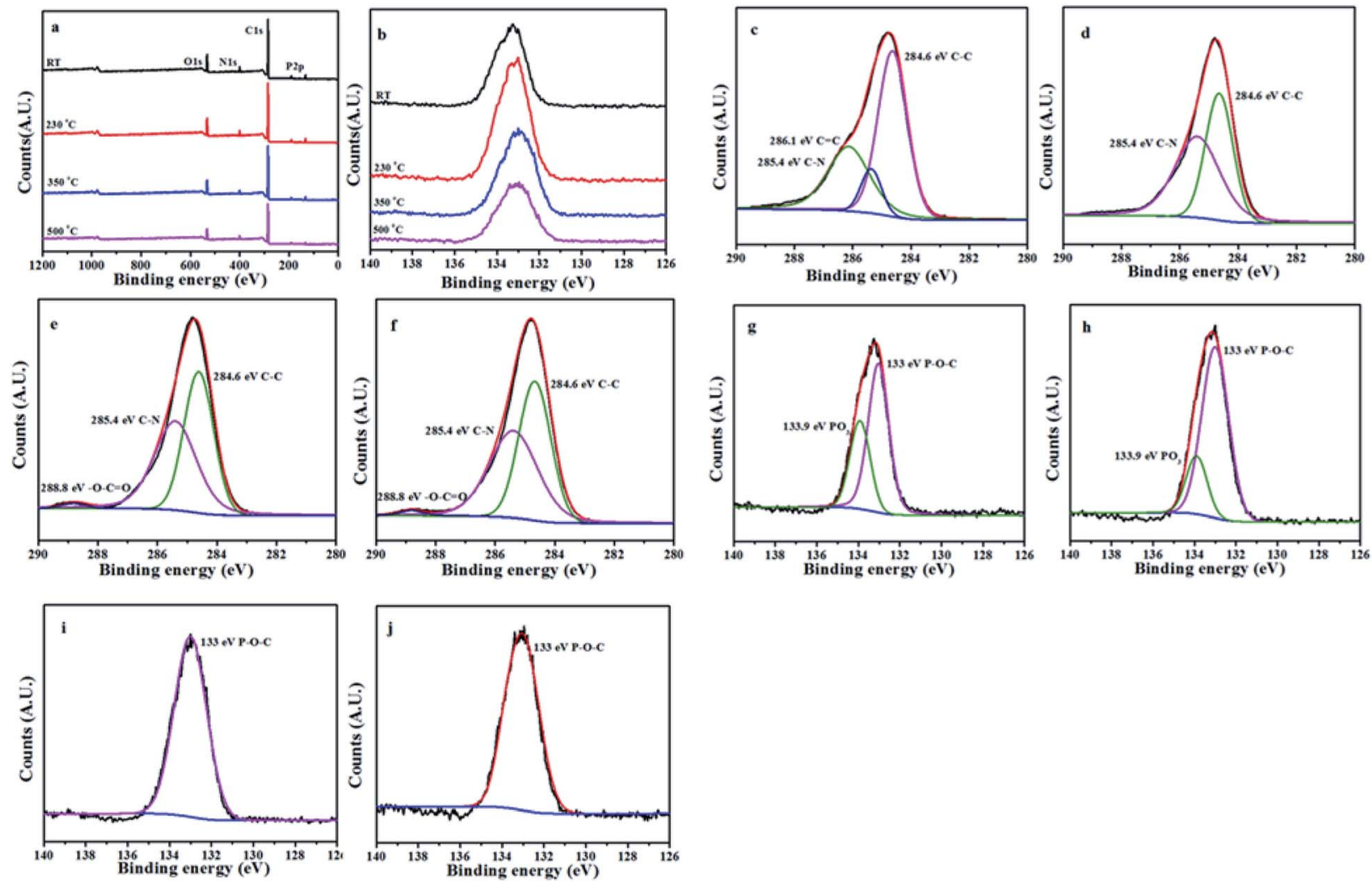

Fig. 5 XPS spectra of L15HD at different temperature (a); P2p XPS spectra of L15HD at different temperature (b); C1s XPS spectra at room temperature (c); C1s XPS spectra at $230{ }^{\circ} \mathrm{C}$ (d); C1s XPS spectra of at $350{ }^{\circ} \mathrm{C}$ (e); C1s XPS spectra at $500{ }^{\circ} \mathrm{C}$ (f); P2p XPS spectra at room temperature (g); P2p XPS spectra at $230{ }^{\circ} \mathrm{C}$ (h); P2p XPS spectra at $350{ }^{\circ} \mathrm{C}$ (i); P2p XPS spectra at $500^{\circ} \mathrm{C}(\mathrm{j})$. 
Table 3 XPS data of L15HD at different temperature

\begin{tabular}{lcccc}
\hline & \multicolumn{4}{l}{ Temperature $\left({ }^{\circ} \mathrm{C}\right)$} \\
\cline { 2 - 5 } Peak & $\mathrm{RT}$ & 280 & 380 & 500 \\
\hline C1s & 74.45 & 76.17 & 78.96 & 78.94 \\
O1s & 18.9 & 15.25 & 13.58 & 13.4 \\
N1s & 4.25 & 4.79 & 4.01 & 4.88 \\
P2p & 2.4 & 3.80 & 3.45 & 2.79 \\
\hline
\end{tabular}

example. The XPS measurement of the L15HD at different degradation step temperature: RT, 230, 350 and $500{ }^{\circ} \mathrm{C}$ were performed and analysed in detail. The XPS spectra of L15HD treated at different temperature are shown in Fig. 5, and the element content data are summarized in Table 3.

It can be observed from Fig. $5 \mathrm{a}$ and $\mathrm{b}$ and Table 3 that the phosphorus content increased first and then decreased with the increasing temperature. The initial decomposition of phosphate was in the solid phase. With the increase of temperature, part of the $\mathrm{PO}^{*}$ ions would volatilize into gas phase. The C1s spectra was shown in Fig. 5c-f. The peak at around $284.6 \mathrm{eV}$ was attributed to $\mathrm{C}-\mathrm{C}$ in aliphatic and aromatic species, ${ }^{36-38}$ the peak at around $285.4 \mathrm{eV}$ was assigned as $\mathrm{C}-\mathrm{N}$ bonds in the HDI acting as bridges, ${ }^{36}$ the peak at around $286.1 \mathrm{eV}$ was assigned to $\mathrm{C}=\mathrm{C}$ in aromatic ring structures. ${ }^{39}$ In addition, as shown in Fig. 5e and $\mathrm{f}$, a new peak appeared at around $288.8 \mathrm{eV}$ which was attributed to $-\mathrm{C}(=\mathrm{O})-\mathrm{O}-\mathrm{R}$ groups. ${ }^{37}$ This may be due to the esterification reaction of the hydroxyl group produced in the decomposition with the phosphoric acid produced from L15HD to form a $\mathrm{P}-\mathrm{O}-\mathrm{C}$ crosslinked structure. ${ }^{37,40}$ As shown in the $\mathrm{P} 2 \mathrm{p}$ XPS spectra of Fig. $5 \mathrm{~g}-\mathrm{j}$, the peaks at around $133.0 \mathrm{eV}$ and 133.9 eV were assigned as $\mathrm{P}-\mathrm{O}-\mathrm{C}$ and $\mathrm{PO}_{3}$ groups, respectively. ${ }^{36}$ It further illustrated that the decomposition of flame retardant produced phosphate and $\mathrm{P}-\mathrm{O}-\mathrm{C}$ structure. And the peaks at $133.9 \mathrm{eV}$ in Fig. $5 \mathrm{i}$ and j disappeared, which indicated that there was no phosphate left in the carbon residue.

\section{Properties of the flame-retarding LPU (FLPU)}

Flame-retardant properties of FLPU. The properties of flame retardancy were investigated by limiting oxygen index (LOI). The results of LOI tests are presented in Table 4 . The LOI value of pure PU was only $17.1 \%$, and the LOI of $\mathrm{L}_{20} \mathrm{PU}(20 \%$ lignin in LPU) was $21.3 \%$ with no dripping, which means the lignin could be flame retardant of PU.

When $15 \%$ prepared flame retardant L5HD (5\% lignin in LHD) was inserted in $\mathrm{L}_{20} \mathrm{PU}$, the LOI value of FLPU was further increased to be $25.3 \%$. This meant LHD was excellent flame retardant for LPU. It was shown the LOI value of FLPU increased with the lignin content in LHDs. When the addition content of LHD was $15 \%$, the LOI value increased from $25.3 \%$ to $28.9 \%$ with the lignin content in LHD increased from 5\% to 15\%. This indicated that lignin in LHDs were effective to improve the flame retardancy of LPU.

With the further increase of loading content of LHD, the LOI values of PU continued to increase. The highest LOI value of $30.2 \%$ appeared in the $\mathrm{F}_{\mathrm{L} 15-25} \mathrm{~L}_{20} \mathrm{PU}$ with $25 \%$ of L15HD inserted
Table 4 TGA and LOI value characterization of the LPU-LHD ${ }^{a}$

\begin{tabular}{lllll}
\hline Samples & LOI $(\%)$ & $T_{5}\left({ }^{\circ} \mathrm{C}\right)$ & $T_{10}\left({ }^{\circ} \mathrm{C}\right)$ & $\begin{array}{l}\text { Residue at } \\
600{ }^{\circ} \mathrm{C}(\%)\end{array}$ \\
\hline $\mathrm{PU}$ & 17.1 & 299 & 314 & 2.48 \\
$\mathrm{~L}_{20} \mathrm{PU}$ & 21.3 & 266 & 295 & 19.92 \\
$\mathrm{~F}_{\mathrm{L} 5-15} \mathrm{~L}_{20} \mathrm{PU}$ & 25.3 & 263 & 297 & 19.55 \\
$\mathrm{~F}_{\mathrm{L} 10-15} \mathrm{~L}_{20} \mathrm{PU}$ & 28.3 & 268 & 290 & 11.87 \\
$\mathrm{~F}_{\mathrm{L} 15-15} \mathrm{~L}_{20} \mathrm{PU}$ & 28.9 & 265 & 290 & 17.98 \\
$\mathrm{~F}_{\mathrm{L} 15-20} \mathrm{~L}_{20} \mathrm{PU}$ & 29.1 & 257 & 287 & 18.21 \\
$\mathrm{~F}_{\mathrm{L} 15-25} \mathrm{~L}_{20} \mathrm{PU}$ & 30.2 & 254 & 286 & 21.01
\end{tabular}

${ }^{a} T_{5}$ : initial degradation temperature (temperature at $5 \%$ weight loss); $T_{10}$ : temperature $10 \%$ weight loss occurs. $\mathrm{F}_{\mathrm{L} 15-25} \mathrm{~L}_{20} \mathrm{PU}$ : content of lignin in the flame-retardant lignin-based polyurethane is $20 \%$, content of lignin in the LHD is $15 \%$, and the mass fraction of the LHD is $25 \%$.

in $\mathrm{L}_{20} \mathrm{PU}$. On the one hand, as an abundant polyphenol biopolymeric material with substantial aromatic structures, lignin can promote the formation of carbon layer and had a certain degree of flame retardancy. ${ }^{22,41}$ In addition, phosphoruscontaining flame retardant LHD produced phosphate compounds during the decomposition process, which promoted the char forming of the soft segments. ${ }^{42}$ And then, a continuous and dense expanded carbon layer was formed on the surface of the FLPU. This could effectively prevent the internal and external combustible gases, oxygen and heat to be transferred. It is worth noting that the decomposition of FLPU produced combustible products, which could be entrapped in the interior swelling carbon layer. This helped to improve the flame retardancy of FLPU. The proposed flame retardancy mechanism of FLPU is shown in Fig. 6. As a result, the flame retardancy of LHD was improved. And thus, the flame retardant property of FLPU was improved with the increasing content of LHDs. Furthermore, all of the FLPUs in this study did not show dripping during burning. The results evidenced that lignin has a significant char formation and synergistic flame retardancy effect for LHDs and FLPU.

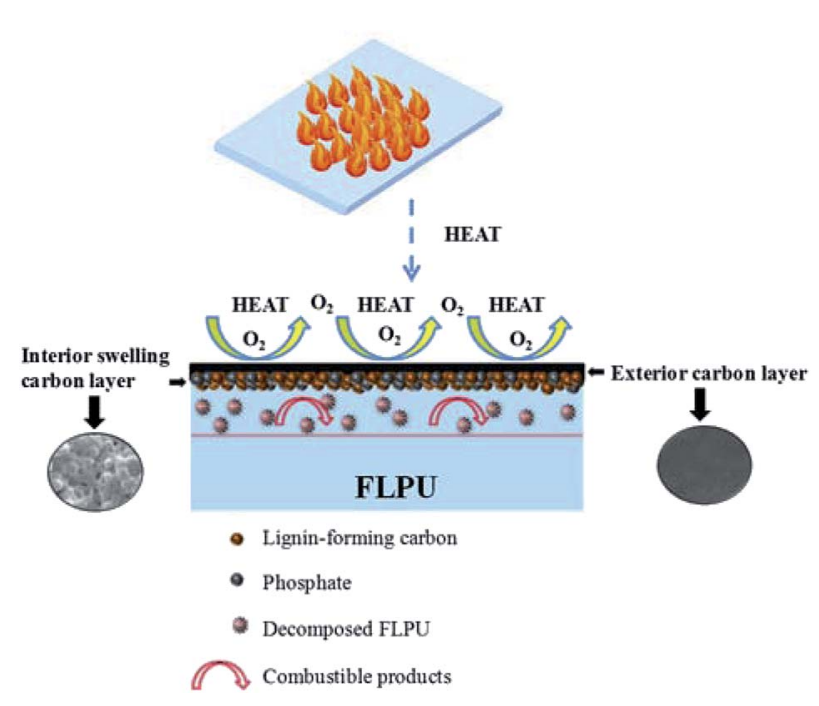

Fig. 6 Flame-retardancy mechanism for FLPU. 


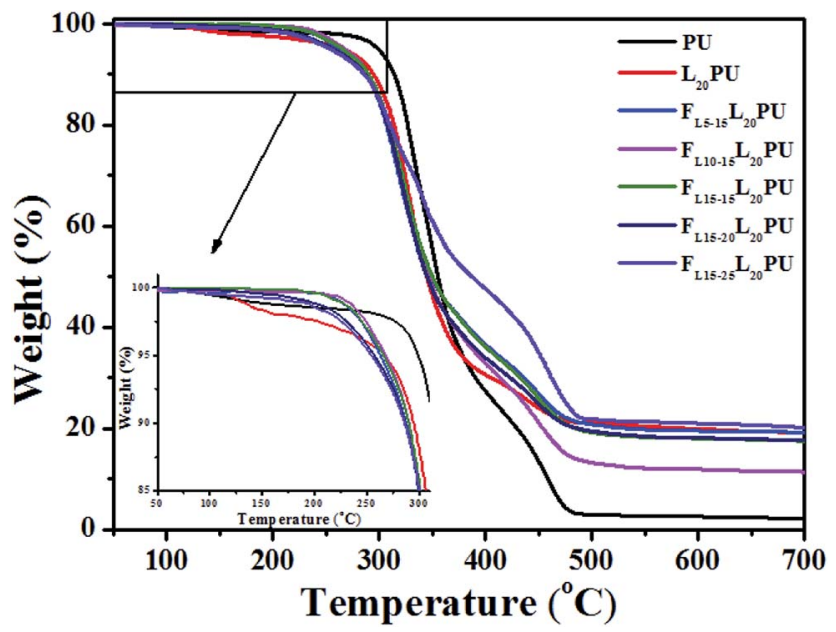

Fig. 7 TGA curves of FLPU films.

Thermal stability of FLPU films. The influence of lignin content on LHDs and the content of LHDs on the thermal stability of LPU films was studied by the thermogravimetric analysis. The TGA curves of the FLPU films are shown in Fig. 7. Some important data, including the $5 \%$ mass loss temperature $\left(T_{5}\right)$, the $10 \%$ mass loss temperature $\left(T_{10}\right)$ and the residue at 600 ${ }^{\circ} \mathrm{C}$ are listed in Table 4. According to the results of TGA as shown in Fig. 7, all the samples showed two-step degradation processes in addition to $\mathrm{PU}$ and $\mathrm{L}_{20} \mathrm{PU}$. PU and $\mathrm{L}_{20} \mathrm{PU}$ decomposed first between $130-240{ }^{\circ} \mathrm{C}$. According to the TGA result of lignin, this degradation stage was related to the decomposition of lignin and the loss of moisture. This was because a small fraction of lignin in $\mathrm{L}_{20} \mathrm{PU}$ did not completely react with isocyanate, and the excess -NCO was reacted with amide hydroxyl to form urea linkages leading to unstable LPU. ${ }^{31}$ However, FLPU had better thermal stability without decomposition at this temperature. This showed that LHD can promote the crosslinking reaction of free lignin that did not fully react in LPU during the curing of polyurethane. This led to a higher crosslinking degree and a more stable network structure of the FLPU.

As shown in Fig. 7, the first degradation step $\left(260\right.$ to $\left.390{ }^{\circ} \mathrm{C}\right)$ of FLPU was a degradation of hard segment duo to the pyrolysis of carbamate structure. ${ }^{34,42-44}$ The second degradation step (420 to 490 ${ }^{\circ} \mathrm{C}$ ) was a degradation of soft segment. As presented in Table 4, the $5 \%$ weight loss degradation temperature $\left(T_{5}\right)$ of $\mathrm{PU}$ and $\mathrm{L}_{20} \mathrm{PU}$ was found to be at $299{ }^{\circ} \mathrm{C}$ and $266{ }^{\circ} \mathrm{C}$, respectively. The lower $T_{5}$ of FLPU was because lignin decomposed at lower temperature. On the other hand, with the addition of LHD in $\mathrm{L}_{20} \mathrm{PU}$ matrix, the $T_{5}$ of FLPU films decreased gradually, which may be attributed to the fact that the thermal stability of LHDs were lower than that of $\mathrm{L}_{20} \mathrm{PU}$. It was reasonable that, as flame retardant, the decomposition of LHDs occurred prior to the $\mathrm{L}_{20} \mathrm{PU}$ when the samples were heated, because the flame retardants were easier to degrade to accelerate carbonization of the matrix..$^{21,45}$
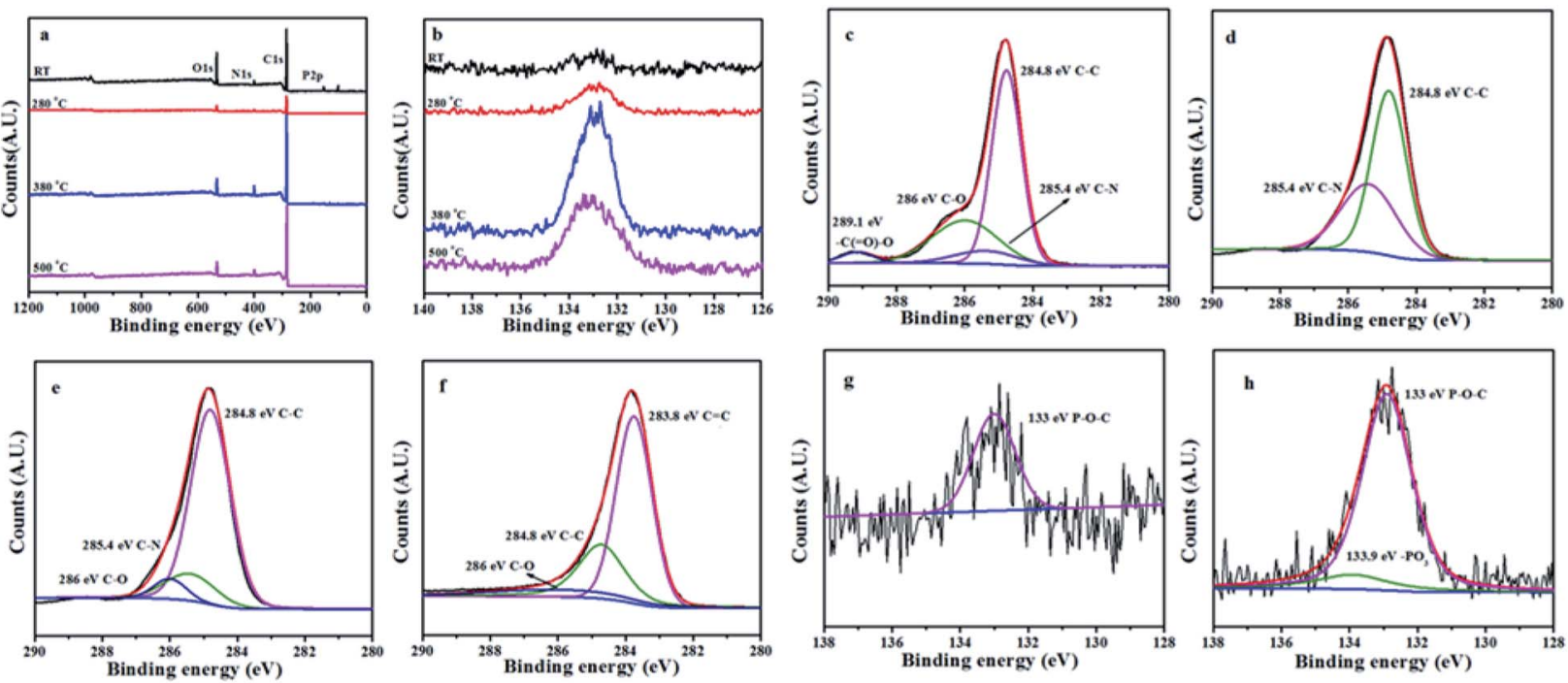
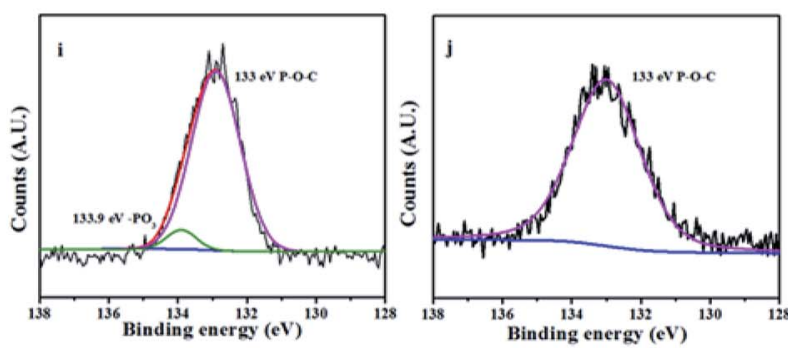

Fig. 8 XPS spectra scan of $F_{L 15-25} L_{20} P U$ at different temperature (a); P2p XPS spectra of $F_{L 15-25} L_{20} P U$ at different temperature (b); $C 1 s$ XPS spectra at room temperature (c); C1s XPS spectra at $280{ }^{\circ} \mathrm{C}$ (d); C1s XPS spectra at $380{ }^{\circ} \mathrm{C}$ (e); C1s XPS spectra at $500{ }^{\circ} \mathrm{C}$ (f); P2p XPS spectra at room temperature (g); P2p XPS spectra at $280{ }^{\circ} \mathrm{C}$ (h); P2p XPS spectra at $380{ }^{\circ} \mathrm{C}$ (i); P2p XPS spectra at $500{ }^{\circ} \mathrm{C}(\mathrm{j})$. 
Table 5 XPS data of $\mathrm{FL}_{15-25} \mathrm{~L}_{20} \mathrm{PU}$ at different temperature

\begin{tabular}{lrrrr}
\hline & \multicolumn{4}{l}{ Temperature $\left({ }^{\circ} \mathrm{C}\right)$} \\
\cline { 2 - 5 } Peak & \multicolumn{1}{l}{ RT } & 280 & 380 & \multicolumn{1}{c}{500} \\
\hline C1s & 77.63 & 78.89 & 83.5 & 85.44 \\
O1s & 18.79 & 13.46 & 9.13 & 8.26 \\
N1s & 3.23 & 6.52 & 6.22 & 5.42 \\
P2p & 0.35 & 1.13 & 1.14 & 0.89
\end{tabular}

Furthermore, LHDs produced phosphate compounds during the degradation which promoted the char forming. Therefore, the char residues of FLPU films increased with the increase of flame retardant LHDs content. The residue char content reached $21.01 \%$, while that of PU was only $2.48 \%$. The TGA results proved that the inserted L15HD could enhance the flame retardancy by increasing the char yield.

\section{XPS test of $\mathbf{F}_{\mathrm{L15-25}} \mathbf{L}_{20} \mathrm{PU}$}

The $\mathrm{F}_{\mathrm{L15-25}} \mathrm{L}_{20} \mathrm{PU}$ sample was treated in a muffle furnace under a nitrogen atmosphere at various temperatures. The XPS measurement of the $\mathrm{F}_{\mathrm{L} 15-25} \mathrm{~L}_{20} \mathrm{PU}$ at different temperature ranges: RT, 280, 380 and $500{ }^{\circ} \mathrm{C}$ were performed and analysed in detail. The XPS spectra of $\mathrm{F}_{\mathrm{L} 15-25} \mathrm{~L}_{20} \mathrm{PU}$ at different temperature are shown in Fig. 8, and the element content data are illustrated in Table 5.

As shown in Table 5, the content of phosphorus also increased first and then decreased as the temperature increased, and was $0.89 \%$ at $500{ }^{\circ} \mathrm{C}$. It can be seen from Fig. $8 \mathrm{c}-\mathrm{f}$, the C1s spectra of $\mathrm{F}_{\mathrm{L} 15-25} \mathrm{~L}_{20} \mathrm{PU}$, the peak at around $284.8 \mathrm{eV}$ was attributed to $\mathrm{C}-\mathrm{C}$ in aliphatic and aromatic species, ${ }^{39}$ the peak at around $285.4 \mathrm{eV}$ was assigned to $\mathrm{C}-\mathrm{N}$ bonds in FLPU. The peaks at around $286.0 \mathrm{eV}$ and $289.1 \mathrm{eV}$ were corresponded to $\mathrm{C}-\mathrm{O},{ }^{49,50}$ and $-\mathrm{C}(=$ O)-O-R bonding, ${ }^{46}$ respectively. Another new peak at $283.8 \mathrm{eV}$ was assigned to $\mathrm{C}=\mathrm{C}$ in aromatic structures. ${ }^{47}$ As shown in Fig. $8 \mathrm{~g}-\mathrm{j}$, the P2p XPS spectra, the peak around $133.0 \mathrm{eV}$ and $133.9 \mathrm{eV}$ were assigned to $\mathrm{P}-\mathrm{O}-\mathrm{C}$ and $\mathrm{PO}_{3}$ groups, respectively. ${ }^{36}$ In Fig. $8 \mathrm{~g}$ and $\mathrm{j}$, the peak at $133.0 \mathrm{eV}$ was attributed as $\mathrm{P}-\mathrm{O}-\mathrm{C}$ bonds in L15HD and char residue. In Fig. $8 \mathrm{~h}$ and i, the double peak at $133.0 \mathrm{eV}$ and $133.9 \mathrm{eV}$ were assigned to the phosphate produced by the decomposition of L15HD and the cross-linked structure of $\mathrm{P}-\mathrm{O}-\mathrm{C}$. The formation of these carbon layers can compact the surface and improve the flame retardancy of FLPU.

\section{Morphology and structure of residual char}

The char residue surface and inner morphology of thermally treated LPU and FLPU samples were characterized by SEM. Fig. 9 shows the microstructures of interior and exterior char of PU and LPU formed in the limiting oxygen index test. From Fig. 9, there were lots of cracks on the outer char of pure PU, which made the char couldn't stop further combustion. On the other hand, its inner char was incompact and empty, which suggested its intrinsic poor flame retardancy. However, the carbon layer became continuous and dense after being incorporated with flame retardant LHDs. The expansion carbon layer was so continuously densified that it could protect the underlying
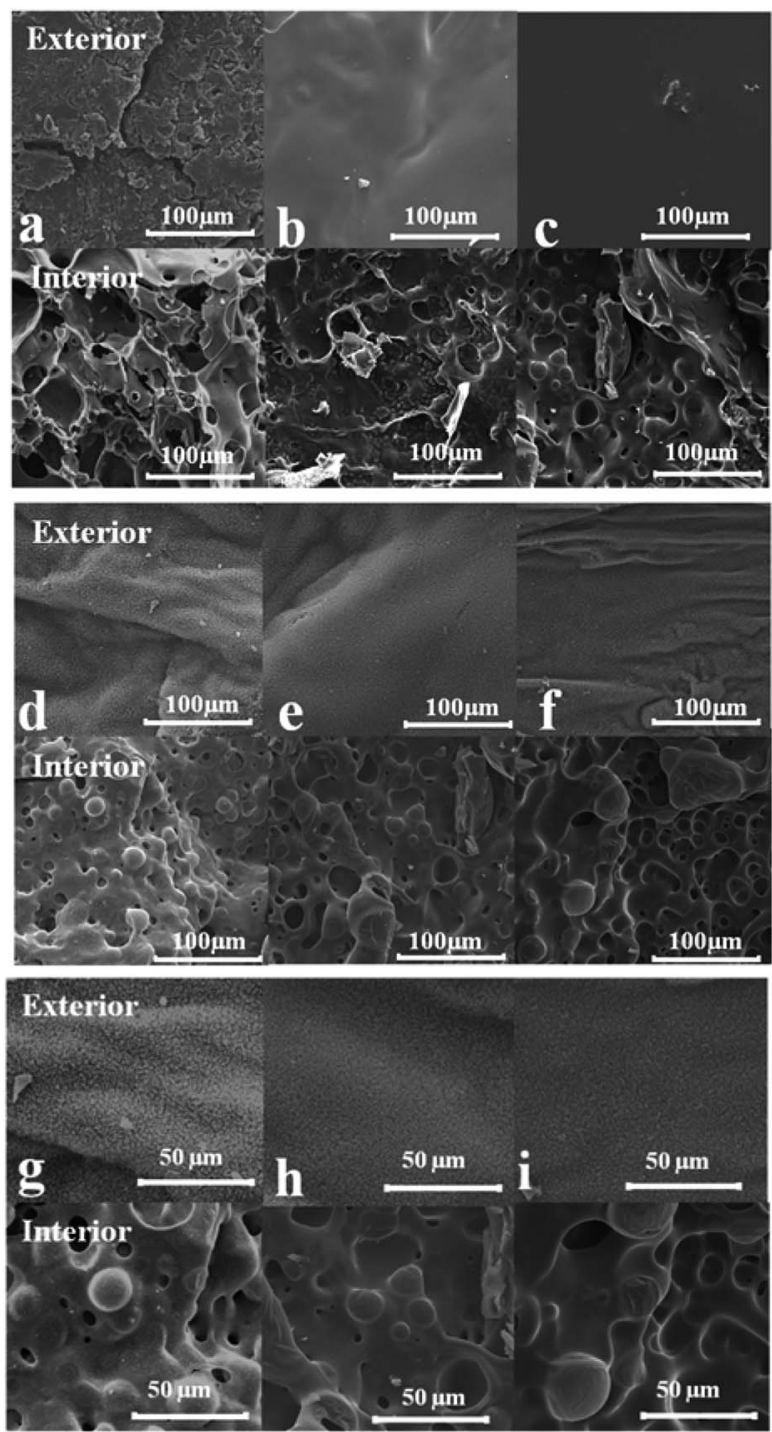

Fig. 9 SEM of exterior and interior surfaces of the char residue after

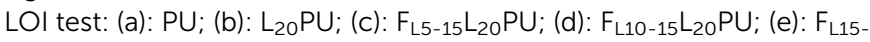
${ }_{15} \mathrm{~L}_{20} \mathrm{PU}$; (f): $F_{\mathrm{L} 15-25} \mathrm{~L}_{20} \mathrm{PU} ;(\mathrm{g}) /(\mathrm{h}) /(\mathrm{i})$ were the enlarged view of $(\mathrm{d}) /(\mathrm{e}) /(\mathrm{f})$.

polymer. The charring layer played a very important role in preventing the heat transfer, flame spreading, and droplet which were generated during the combustion process. ${ }^{40}$ As shown in Fig. 9d-f, the burnt surface had plenty of rugged creases, and the inner surface presented a large number of bubbles. Fig. $9 g-i$ gave the enlarged view of FLPU for comparison. It can be more clearly observed the denseful structure of the inner and outer carbon layers. This intumescent group could slow down the heat and mass transfer between gas and the solidified phase. ${ }^{21}$ It is obvious that the compact layer was a excellent flame shield to protect the underlying composite. This could obviously improve the flame retardancy of FLPU films. This SEM characterization result was consistent with that of LOI test.

\section{Coating properties of the FLPU films}

Table 6 shows the coating properties of the FLPU films. The hardness of the coatings is critical because the wear resistance 
Table 6 Coating properties of the FLPU film

\begin{tabular}{|c|c|c|c|c|}
\hline Samples & Hardness & Flexibility $(\mathrm{mm})$ & Adhesion & Impact resistance $(100 \mathrm{~cm} / 1000 \mathrm{~g})$ \\
\hline PU & B & 2 & 0 & Pass \\
\hline $\mathrm{L}_{20} \mathrm{PU}$ & $3 \mathrm{H}$ & 2 & 0 & Pass \\
\hline $\mathrm{F}_{\mathrm{L} 10-15} \mathrm{~L}_{20} \mathrm{PU}$ & $3 \mathrm{H}$ & 2 & 0 & Pass \\
\hline $\mathrm{F}_{\mathrm{L} 15-15} \mathrm{~L}_{20} \mathrm{PU}$ & $4 \mathrm{H}$ & 2 & 0 & Pass \\
\hline $\mathrm{F}_{\mathrm{L} 15-20} \mathrm{~L}_{20} \mathrm{PU}$ & $4 \mathrm{H}$ & 2 & 0 & Pass \\
\hline
\end{tabular}

and scratch resistance of the coatings are affected by their hardness. ${ }^{48,49}$ The flexibility of the molecular chain and the crosslink density of the polymer network can affect the hardness. ${ }^{50}$ As can be seen from Table 6 , with the addition of lignin, the hardness of the PU film was increased from grade $\mathrm{B}$ to $3 \mathrm{H}$. This was because the addition of lignin can form a crosslinked structure in polyurethane and increase the crosslink density. The addition of LHD further increased the hardness of PU up to $4 \mathrm{H}$. This was mainly due to the addition of LHD into the films. The interaction between the flame retardants LHD and LPU matrix was strong because of the co-existence of lignin and cocuring of LHD and LPU.

The hydroxyl groups in the polymer chain can interact with the substrate. Therefore, the content of hydroxyl groups in the polymer can affect the adhesion of the polymer to the substrate. ${ }^{51}$ As can be seen from Table 6, all samples exhibited excellent flexibility, adhesion, and impact resistance. The film's flexibility was up to $2 \mathrm{~mm}$, adhesion was 0 , impact resistance passed the CJQ-II film impactor $100 \mathrm{~cm} / 1000 \mathrm{~g}$ test. The flexibility of the flame-retarded lignin-based PU film was mainly maintained due to the presence of some aliphatic side chains in HDI and the lignin structure. ${ }^{\mathbf{5}}$

Therefore, the mechanical properties of the FLPU film can be reinforced by LHD because both LHD and PU contained the same urethane structure and the phenyl groups of lignin. This was shown to be an effective way for solving the compatibility between the flame retardant and the matrix PU or lignin based PU. Thus, the FLPU could contain not only the flame retardant element phosphorus but also the lignin, which could act as the reinforcing agent and carbonizing agent. Its performances in terms of thermal stability, flame retardancy and properties as coatings were much improved with LHD insertion.

\section{Conclusions}

Lignin-based flame retardant was successfully synthesized in this study, and its chemical structure was confirmed by FTIR, ${ }^{1} \mathrm{H}$ NMR and ${ }^{31} \mathrm{P}$ NMR. The temperature of $5 \%$ mass loss of LHDs with different lignin content was higher than $242{ }^{\circ} \mathrm{C}$, which showed excellent thermal stability. And with the increase of lignin content, the residual char of LHD were increased to be as high as $17.54 \%$ at $500{ }^{\circ} \mathrm{C}$. LHDs were compounded with lignin-based polyurethanes to obtain FLPU, and the LOI test, TGA analysis, and SEM were performed to investigate the combustion properties and thermal stability of FLPU. The results showed that the LOI value of the flame-retardant polyurethane reached $30.2 \%$ when the $\mathrm{L} 15 \mathrm{HD}$ content reached $25 \%$ in $\mathrm{L}_{20} \mathrm{PU}$, illustrating excellent flame-retardant properties. The lignin-based flame retardant was beneficial to the formation of the expanded carbon layer, and could play a key role in the flame retardation of the solidified phase. FLPU also showed excellent properties for coating applications. The hardness of FLPU films reached $4 \mathrm{H}$. And the flexibility was up to $2 \mathrm{~mm}$. The adhesion was 0 , and the impact resistance passed the CJQ-II film impactor $100 \mathrm{~cm} / 1000 \mathrm{~g}$ test. Thus, LHD could be promising to be applied as flame retardant and reinforcing material for LPU. More importantly, the prepared lignin-based flame retardant could also be applied in other materials, such as fully biodegradable flame-retardant polylactic acid composites.

\section{Conflicts of interest}

There are no conflicts to declare.

\section{Acknowledgements}

This work was financially supported by the National Natural Science Foundation of China [No. 21404013], the Science and Technology Development Plan of Jilin Province, China [No. 20160101323JC, 20170101110JC, 20180201076GX, 20180201075GX], the Jilin Provincial Development and Reform Commission, China [2018C041-1], the Open Research Fund of State Key Laboratory of Polymer Physics and Chemistry, Changchun Institute of Applied Chemistry, Chinese Academy of Sciences.

\section{References}

1 C. O. Tuck, E. Pérez, I. T. Horváth, R. A. Sheldon and M. Poliakoff, Science, 2012, 337(6095), 695-699.

2 S. Sen, S. Patil and D. S. Argyropoulos, Green Chem., 2015, 17(11), 4862-4887.

3 B. M. Upton and A. M. Kasko, Chem. Rev., 2016, 116(4), 22752306.

4 F. G. Calvo-Flores and J. A. Dobado, Chemsuschem, 2010, 3(11), 1227-1235.

5 H. Chung and N. R. Washburn, ACS Appl. Mater. Interfaces, 2012, 4(6), 2840-2846.

6 W. Liu, R. Zhou, H. L. S. Goh, S. Huang and X. Lu, ACS Appl. Mater. Interfaces, 2014, 6(8), 5810-5817. 
7 T. Saito, R. H. Brown, M. A. Hunt, D. L. Pickel, J. M. Pickel, J. M. Messman, F. S. Baker, M. Keller and A. K. Naskar, Green Chem., 2012, 14(12), 3295-3303.

8 R. Auvergne, S. Caillol, G. David, B. Boutevin and J. P. Pascault, Chem. Rev., 2014, 114(2), 1082-1115.

9 G. Sun, H. Sun, Y. Liu, B. Zhao, N. Zhu and K. Hu, Polymer, 2007, 48(1), 330-337.

10 C. Vilela, A. F. Sousa, A. C. Fonseca, A. C. Serra, J. F. J. Coelho, C. S. R. Freire and A. J. D. Silvestre, Polym. Chem., 2014, 5(9), 3119-3141.

11 N. D. Luong, N. T. T. Binh, D. D. Le, O. K. Dong, D. S. Kim, D. S. Kim, S. H. Lee, J. K. Baek, Y. S. Lee and J. D. Nam, Polym. Bull., 2012, 68(3), 879-890.

12 Y. Kang, Z. Chen, B. Wang and Y. Yang, Ind. Crops Prod., 2014, 56(56), 105-112.

13 B. L. Xue, J. L. Wen, M. Q. Zhu and R. C. Sun, RSC Adv., 2014, 4(68), 36089-36096.

14 B. L. Xue, J. L. Wen and R. C. Sun, ACS Sustainable Chem. Eng., 2014, 2(6), 1474-1480.

15 H. Lim, eXPRESS Polym. Lett., 2008, 2(3), 194-200.

16 L. Song, Y. Hu, Y. Tang, R. Zhang, Z. Chen and W. Fan, Polym. Degrad. Stab., 2005, 87(1), 111-116.

17 C. K. Ranaweera, M. Ionescu, N. Bilic, X. Wan, P. K. Kahol and R. K. Gupta, J. Renewable Mater., 2017, 5(suppl. 1), 1-12.

18 H. B. Chen, P. Shen, M. J. Chen, H. B. Zhao and D. A. Schiraldi, ACS Appl. Mater. Interfaces, 2016, 8(47), 32557-32564.

19 W. M. Lu, Q. Li, Y. Zhang, H. W. Yu, S. Hirose, H. Hatakeyama, Y. Matsumoto and Z. F. Jin, J. Wood Sci., 2018, 64(3), 287-293.

20 L. P. Gao, G. Y. Zheng, Y. H. Zhou, L. H. Hu and G. D. Feng, J. Therm. Anal. Calorim., 2015, 120(2), 1311-1325.

21 H. B. Zhu, Z. M. Peng, Y. M. Chen, G. Y. Li, L. Wang, R. Pang, Z. U. H. Khan and P. Wan, RSC Adv., 2014, 4(98), 5527155279.

22 A. D. Chirico, M. Armanini, P. Chini, G. Cioccolo, F. Provasoli and G. Audisio, Polym. Degrad. Stab., 2003, 79(1), 139-145.

23 L. Costes, F. Laoutid, M. Aguedo, A. Richel, S. Brohez, C. Delvosalle and Ph. Dubois, Eur. Polym. J., 2016, 84, 652667.

24 B. Prieur, M. Meub, M. Wittemann, R. Klein, S. Bellayer, G. Fontaine and S. Bourbigot, Polym. Degrad. Stab., 2016, 127, 32-43.

25 C. H. Lin, C. C. Feng and T. Y. Hwang, Eur. Polym. J., 2007, 43(3), 725-742.

26 P. Wang, F. Yang, L. Li and Z. Cai, Polym. Degrad. Stab., 2016, 129, 156-167.

27 N. Mandlekar, A. Cayla, F. Rault, S. Giraud, F. SalaÜn, G. Malucelli and J. P. Guan, An Overview on the Use of Lignin and Its Derivatives in Fire Retardant Polymer Systems, In Tech Open, 2018.
28 S. Gaan, S. Liang, H. Mispreuve, H. Perler, R. Naescher and M. Neisius, Polym. Degrad. Stab., 2015, 113, 180-188.

29 Z. Jia, C. Lu, P. Zhou and L. Wang, RSC Adv., 2015, 5(66), 53949-53955.

30 M. Brebu and C. Vasile, Cellul. Chem. Technol., 2010, 44(9), 353-363.

31 Z. M. Wang, X. H. Yang, Y. H. Zhou and C. G. Liu, BioResources, 2013, 8(3), 3833-3843.

32 W. Fiddler, W. E. Parker, A. E. Wasserman and R. C. Doerr, J. Agric. Food Chem., 1967, 15(5), 757-761.

33 C. Branca, A. Paola Giudicianni and C. D. Blasi, Ind. Eng. Chem. Res., 2003, 42(14), 3190-3202.

34 N. M. Neisius, M. Lutz, D. Rentsch, P. Hemberger and S. Gaan, Ind. Eng. Chem. Res., 2014, 53(8), 2889-2896.

35 K. Srikulkit, C. Iamsamai and S. T. Dubas, J. Met., Mater. Miner., 2006, 16(2), 41-45.

36 X. L. Chen, Y. Hu, C. M. Jiao and L. Song, Polym. Degrad. Stab., 2007, 92(6), 1141-1150.

37 W. C. Zhang, X. M. Li and R. J. Yang, Polym. Degrad. Stab., 2014, 99(1), 118-126.

38 W. C. Zhang, X. M. Li and R. J. Yang, Polym. Degrad. Stab., 2011, 96(10), 1821-1832.

39 Z. H. Zheng, Y. Liu, L. Zhong and H. Y. Wang, J. Mater. Sci., 2016, 51(12), 5857-5871.

40 Z. H. Zheng, Y. Liu, L. Zhang, B. Y. Dai, X. D. Yang and H. Y. Yan, J. Therm. Anal. Calorim., 2017, 127(3), 2013-2023.

41 B. Prieur, M. Meub, M. Wittemann, R. Klein, S. Bellayer, G. Fontaine and S. Bourbigot, RSC Adv., 2017, 7, 1686616877.

42 R. Yang, W. Hu, L. Xu, Y. Song and J. Li, Polym. Degrad. Stab., 2015, 122, 102-109.

43 W. Zhou, F. Chen, H. Zhang and J. Wang, J. Wood Chem. Technol., 2017, 37(5), 323-333.

44 D. K. Chattopadhyay and D. C. Webster, Prog. Polym. Sci., 2009, 34(10), 1068-1133.

45 H. Ma, L. Tong, Z. Xu, Z. Fang, Y. Jin and F. Lu, Polym. Degrad. Stab., 2007, 92(4), 720-726.

46 O. Ivashenko, J. T. van Herpt, B. L. Feringa, W. R. Browne and P. Rudolf, Chem. Phys. Lett., 2013, 559(2), 76-81.

47 W. Zhu, X. L. Cai, Z. W. Xu, L. Zhou and Z. Y. Wang, Integr. Ferroelectr., 2017, 180(1), 1-11.

48 J. Sun, X. Wang and D. Wu, ACS Appl. Mater. Interfaces, 2012, 4(8), 4047-4061.

49 R. Schwalm, L. Häußling, W. Reich, E. Beck, P. Enenkel and K. Menzel, Prog. Org. Coat., 1997, 32(1-4), 191-196.

50 G. Bayramoğlu, M. V. Kahraman, N. Kayaman-Apohan and A. Güngör, Prog. Org. Coat., 2006, 57(1), 50-55.

51 T. H. Chiang and T. E. Hsieh, Int. J. Adhes. Adhes., 2006, 26(7), 520-531.

52 J. Dai, S. Ma, X. Liu, L. Han, Y. Wu, X. Dai and J. Zhu, Prog. Org. Coat., 2015, 78, 49-54. 